\title{
Low selenium status is associated with the occurrence of the pregnancy disease preeclampsia in UK women
}

Margaret P. Rayman, DPhil (Oxon) ${ }^{1}$, Peter Bode, $\mathrm{PhD}^{2}$, Christopher W. G. Redman, $\mathrm{MD}^{3}$

${ }^{1}$ Guildford, Surrey, UK, ${ }^{2}$ Delft, The Netherlands, ${ }^{3}$ Oxford, Oxfordshire, UK

${ }^{1}$ University of Surrey, School of Biomedical and Life Sciences, ${ }^{2}$ Delft University of Technology, Interfaculty Reactor Institute, ${ }^{3}$ University of Oxford, Nuffield Department of Obstetrics and Gynaecology, John Radcliffe Hospital

\section{Financial support}

There were no external sources of financial support. The study was carried out with established personnel and within internal budgets.

\section{Reprints and correspondence}

Dr. Margaret P. Rayman, School of Biomedical and Life Sciences, University of Surrey, Guildford GU2 7XH, UK

Business telephone number (44) 1483686447

Home telephone number $\quad$ (44) 1483562882

Fax number $\quad$ (44) 1483686481

Word count (text) 2490 


\section{Condensation}

Low selenium status in UK pregnant women is significantly associated with a greater risk of occurrence of preeclampsia and more severe expression of the disease. 


\section{Low selenium status is associated with the occurrence of the pregnancy disease preeclampsia in UK women}

Margaret P. Rayman, Peter Bode, Christopher W. G. Redman

OBJECTIVE: As the trace element selenium behaves as an antioxidant and peroxynitrite scavenger when incorporated into selenoproteins, our objective was to determine whether low selenium status was associated with a greater risk of occurrence of preeclampsia.

STUDY DESIGN: 53 preeclamptic patients and 53 matched pregnant controls at the John Radcliffe Hospital, Oxford, gave clippings of their toenails (laid down from 3-12 months previously) for selenium determination by neutron activation analysis. Clinical characteristics of the women and their infants were recorded. Statistical analysis was by Wilcoxon's signed rank test and odds ratios were calculated by the ratio of discordant pairs.

RESULTS: Median toenail selenium concentrations in the preeclamptic subjects were significantly lower than in their matched controls $(P=0.001)$. Being in the bottom tertile of toenail selenium was associated with a 4.4-fold (95\% CI 1.6-14.9) greater incidence of the condition. Within the preeclamptic group, lower selenium status was significantly associated $(P=0.029)$ with more severe expression of disease, as measured by delivery before 32 weeks. CONCLUSION: In the light of the reduction in selenium status in a number of European countries in recent years, this study raises the question of whether a small increase in selenium intake might help prevent preeclampsia in susceptible women.

\section{Key words}

Preeclampsia, pregnancy, selenium, peroxynitrite, selenoprotein $\mathrm{P}$ 
Preeclampsia is a major complication of pregnancy that is associated with high maternal and perinatal morbidity and mortality. It is one of the major indications for elective premature delivery as there is currently no other cure.

Poor placentation is an early preclinical development, the consequences of which are placental ischaemia and oxidative stress. ${ }^{1}$ Blood-borne agents arising from the ischaemic placenta are believed to cause a maternal systemic inflammatory response that includes generalized endothelial-cell dysfunction, which gives rise to the characteristic symptoms of hypertension, proteinuria and sudden oedema., ${ }^{2,3}$ These blood-borne agents, though undefined, are thought likely to be products of placental oxidative stress. ${ }^{3}$ In support of this theory, markers of oxidative-stress have been identified both in the preeclamptic placenta and the maternal circulation ${ }^{4}$, while in a controlled trial, antioxidant supplements (vitamins C and E) were found to reduce the incidence of pre-eclampsia in women at high risk of developing the condition. ${ }^{5}$

The powerful oxidizing and nitrating agent peroxynitrite is a candidate mediator of the endothelial dysfunction of preeclampsia. ${ }^{6}$ There is evidence that peroxynitrite is formed in both the placental and systemic vasculature of women with preeclampsia to a significantly greater extent than in normal pregnancy. ${ }^{6-8}$ The endothelium being a constant source of nitric oxide is a predominant site of peroxynitrite generation when cells generating superoxide are in the vicinity. ${ }^{9}$ Peroxynitrite is a potent inflammatory mediator ${ }^{10}$, causes vasoconstriction, platelet aggregation and thrombus formation ${ }^{11}$ and is capable of interacting with signal transduction pathways linked to vasoactive agents. ${ }^{12}$ It therefore has the capacity, directly or indirectly, to invoke all the pathological changes observed in the vasculature that culminate in endothelial-cell dysfunction. $^{11}$ 
The trace element selenium, in the context of the antioxidant selenoproteins, is capable of limiting adverse endothelial effects. This is because it removes the products of attack by reactive oxygen species (hydroperoxides and oxidized lipoproteins) that can break down to further reactive free radicals and cytotoxic agents ${ }^{13}$ and because selenoprotein $\mathrm{P}$ appears to have a unique capacity to scavenge peroxynitrite at the endothelial surface. ${ }^{10}$ We therefore set out to explore the possible association between selenium status and the occurrence of pre-eclampsia.

To overcome the difficulty experienced in a previous study of the disease process influencing tissue concentrations of selenium ${ }^{14}$, we decided to measure selenium status by using toenail clippings of preeclamptic women and matched pregnant controls. This gives a means of determining selenium concentration before the development of symptoms and even before pregnancy, as toenails are laid down from 3-12 months prior to clipping. ${ }^{15}$

In the current study, toenail selenium was determined by neutron activation analysis, the usual method for such analyses. There is a considerable history of the successful use of toenail selenium concentration as a biomarker of selenium status. ${ }^{15-17}$

\section{Material and methods}

Patients. Fifty-three obstetric patients at the John Radcliffe Hospital, Oxford, were identified as suffering from preeclampsia according to the following criteria:- new hypertension, from below to consistently at or above $90 \mathrm{~mm}$ Hg diastolic pressure; and new proteinuria $\geq 75$ $\mathrm{mg} / \mathrm{mmol}$ creatinine, in the proven absence of urinary-tract infection. Patients included in the group were generally those showing symptoms of preeclampsia at a relatively early gestational age. In Oxford, women who present with preeclampsia after 36 weeks are delivered promptly so 
that there is less time to gain informed consent. Clippings from all ten toenails, free of nail varnish, were collected.

The following clinical characteristics of the patients were recorded:- age, gestational age at time of sampling, systolic and diastolic blood pressure, proteinuria, platelet count, aspartate aminotransferase (AST) activity (an indicator of liver damage), gestational age at the time of delivery, infant sex and birthweight. Infant birthweights were allocated to centiles using “Oxford Standards” charts (Castlemead, Ware UK) allowing correction for length of gestation and sex.

A matched control was selected for each preeclamptic patient from the ante-natal clinic according to the following criteria:- same age \pm 4 years; same gestation \pm 13 days; same parity group namely 0 , 1-3 or 4 or above. Matching for other risk factors was not attempted. Controls had all conceived spontaneously, had never have received a blood transfusion and were not on any medication. Patients and controls were asked if they took any vitamin or mineral supplements, smoked or were vegetarian. The clinical characteristics of the subjects, who were recruited between 1996 and 2001, are recorded in Table 1 .

The Central Oxford Research Ethics Committee approved the study.

Toenail preparation and analysis. Toenails were prepared for analysis according to the protocol developed for hair analysis by the International Atomic Energy Agency. ${ }^{18}$ The toenail samples were placed in acid-washed beakers and washed sequentially with acetone (1 min), distilled deionized water (three washes of $3 \mathrm{~min}$ ) and acetone (1 min) in a sonic bath. If nail varnish was visibly present, the initial acetone wash was continued for a longer time. The samples were placed in a drying oven for two hours at $50^{\circ} \mathrm{C}$, that being the length of time 
required to achieve a constant weight. The dried samples, of typical mass 12 to $135 \mathrm{mg}$, were then weighed into polyethylene capsules ready for analysis.

The selenium content was determined by instrumental neutron activation analysis, using the facilities of the Interfaculty Reactor Institute in Delft. The samples were irradiated for a period of $4 \mathrm{~h}$ in a thermal neutron flux of approximately $5 * 10^{16} \mathrm{~m}^{-2} \mathrm{~s}^{-1}$ together with neutron flux monitors and quality control samples. The radioactivity of the radionuclide ${ }^{75}$ Se was measured via gamma-ray spectroscopy using a well-type Ge-detector. The counting was carried out approximately 20 days after irradiation over a period of $1 \mathrm{~h}$. Quantification was based on the single comparator method. The quality control samples included a blank capsule and a sample of a certified reference material, viz. NIST SRM 1577b Bovine Liver. The selenium concentration, corrected for the contribution of the blank, was determined together with the combined measurement uncertainty covering the most important contributions.

Analysis of NIST SRM 1577b Bovine Liver gave a mean and combined standard uncertainty (45 determinations) of $0.73 \pm 0.005 \mathrm{mgkg}^{-1}$, compared to a certified mean and standard deviation of $0.74 \pm 0.02 \mathrm{mgkg}^{-1}$ demonstrating excellent accuracy of the method. The laboratory has an embedded quality control system for quality assurance and management, which complies with the requirements of the International Standard ISO/IEC 17025:1999 and has been accredited by the Dutch Council for Accreditation since 1993.

Statistical analysis. Since toenail selenium concentrations in the preeclamptic and control matched pairs were positively skewed, they were compared by Wilcoxon’s Signed Rank Test for non-parametric, paired data.

Cases and controls were ranked in tertiles on the basis of toenail selenium. The odds ratio of having preeclampsia for women in the lowest selenium tertile compared to the rest, and 
for women in the top tertile compared to the rest, were calculated by the ratio of discordant pairs. 95\% confidence intervals were calculated by the method of Breslow and Day.

Early delivery ${ }^{19}$ ( $\leq 32$ weeks) and birthweight less than the third centile were selected as indices to test if more severe disease correlated with selenium status. Preeclamptic subjects were divided into tertiles on the basis of toenail selenium. The $\chi^{2}$ test, or Fisher's Exact Test where cell numbers were $<5$, were used to see if there was a significant difference between tertiles with respect to numbers of babies delivered earlier than 32 weeks or birthweight $<3^{\text {rd }}$ centile.

To determine whether selenium was significantly lower in those born before 32 weeks, the pre-eclamptic subjects were divided into two groups according to gestational age at birth (> or $<32$ weeks) and a one-tailed Mann-Whitney U test was applied.

Fisher's Exact test was used to assess whether vegetarianism or the taking of supplements in pregnancy affected selenium concentration.

\section{Results}

The clinical characteristics of the two patient groups are shown in Table I. The preeclamptic women were all suffering from moderately-severe to very-severe disease. Eight had AST levels $>40$ IU/l suggesting a component of liver damage while two had a low platelet count $\left(<100 \times 10^{9}\right.$ cells/l), also indicative of disease severity. Two of the pregnancies were complicated by the HELLP (hemolysis, elevated liver enzymes, low platelets) or ELLP (elevated liver enzymes, low platelets) syndromes. There were six cases of epigastric pain, a symptom of HELLP syndrome. Infants of three women had perinatal, neonatal or postnatal deaths. The control pregnant women gave birth without developing hypertension or proteinuria, to infants 
weighing more than $2500 \mathrm{~g}$, delivered at 37 weeks or later. All control neonates survived normally. All pregnancies were singleton pregnancies.

A scatter-plot of the toenail selenium data from the two groups is shown in Figure 1. There was one clear outlier in the control group. The reason for this is unknown: the subject was not on any supplements. The median of toenail selenium concentrations in the preeclamptic women was significantly lower at $0.56 \mathrm{mg} / \mathrm{kg}$ (interquartile range $0.51-0.64$ ), than in the control women at $0.62 \mathrm{mg} / \mathrm{kg}$ (interquartile range 0.57-0.69; $P<0.001$ ). When all 106 women were ranked in tertiles according to toenail selenium (Table II), it was apparent that almost half the preeclamptic subjects, but only around one eighth of the controls, fell into the bottom tertile. For both groups, the remaining subjects were equally distributed between the middle and top tertiles. This suggests a threshold effect of toenail selenium on risk, with the greatest risk of preeclampsia being associated with the lowest selenium tertile. Odds ratios, calculated so as to take the matched pairing into account, are shown in Table III. The odds of having preeclampsia if in the lowest tertile rather than the middle or upper, are markedly higher, at 4.4:1. The bounds of the 95\% confidence interval (CI, 1.6-14.9) indicate that this is a significant finding.

Further analyses were confined to the preeclampsia group to determine if the severity of the disease correlated with selenium status. The preeclamptic subjects were ranked in tertiles according to toenail selenium. There was no significant difference between the tertiles in terms of the number of babies whose birthweight was less than the third centile. However there were significantly fewer women (one only) in the top tertile who delivered at less than 32 weeks $(P<0.0001)$

When the preeclamptic subjects were divided into two groups according whether they delivered their babies before or after 32 weeks gestation, the 11 women with babies delivered 
before 32 weeks (a measure of disease severity ${ }^{19}$ ) had a significantly lower selenium status than the 42 women who delivered after 32 weeks (median and range, 0.51, 0.35-0.45 mg/kg vs. 0.56, $0.36-0.79 \mathrm{mg} / \mathrm{kg}$ respectively, $P=0.029)$.

The main dietary sources of selenium in the UK are meat, poultry and fish ${ }^{13}$ therefore vegetarians are likely to have a lower intake of selenium. There were only four vegetarians in the pre-eclampsia group and their selenium status was not different from the rest. Neither did the taking of supplements during pregnancy affect selenium status. Too few women in either group smoked (two in the control group and four in the preeclamptic group) to analyze the data with respect to smoking prevalence.

\section{Comment}

The results of this case-control study have shown that for UK pregnant women, being in the bottom tertile of selenium status in the period spanning 3-12 months prior to the development of preeclampsia is associated with a 4.4-fold greater incidence of the condition.

Moreover those preeclamptic women with the lowest selenium status had the most severe disease in terms of the earliest onset, but not in terms of having babies with the lowest birthweight centile. Our findings are consistent with the results of a small supplementation trial carried out in China in women in late pregnancy where $100 \mu \mathrm{g}$ selenium/day was shown to prevent pregnancy-induced hypertension and gestational edema in women deemed to be at risk of pregnancy-induced hypertension. ${ }^{20}$

Since selenium enters the food chain through plants, soil concentration, geochemistry and rainfall largely determine selenium status, which varies considerably throughout the world. ${ }^{13}$ Thus toenail selenium concentrations in this study are similar to those measured in women in the 
Netherlands $[0.575 \pm 0.109 \mathrm{mg} / \mathrm{kg}$ (mean $\pm \mathrm{SD})]^{16}$ but considerably lower than those in US women $[0.83 \pm 0.16-0.92 \pm 0.15 \mathrm{mg} / \mathrm{kg}($ mean $\pm \mathrm{SD})] .{ }^{17}$ The relatively low selenium status observed in this group is consistent with our previous finding of low selenium status in normal obstetric patients, of average gestation 33 weeks, attending the same hospital (median plasma selenium $48.5 \mu \mathrm{g} / \mathrm{l}$, range $23.1-101.4)^{21}$ and the lower selenium status found in most parts of Europe when compared to North America. ${ }^{13}$ A repeat of our study in an area of higher selenium status (e.g. the US where dietary intakes of selenium are higher ${ }^{13}$ ) might not show an association between selenium status and risk of developing preeclampsia.

In our study, the mean toenail selenium among women who smoked (four preeclamptic women and two controls) was lower than the mean in the non-smokers $(0.512 \mathrm{vs} .0 .597 \mathrm{mg} / \mathrm{kg})$ in accordance with previous findings. ${ }^{16}$

There are a number of ways in which selenium in selenoproteins could reduce the risk and severity of preeclampsia. Antioxidant selenoproteins can protect the endothelium by downregulating cytokine-induced adhesion-molecule expression and reducing inflammation. ${ }^{22}$ The family of glutathione peroxidases (GPxs) reduces hydrogen peroxide, lipid and phospholipid hydroperoxides, even when integrated into lipoproteins or membranes. ${ }^{13}$ This prevents their breakdown into further reactive oxygen species and cytotoxic aldehydes, reducing the risk of damage to the endothelium. ${ }^{13}$ Lipid hydroperoxides are also produced by the lipoxygenase and cyclooxygenase pathways leading to prostaglandins, leukotrienes and lipoxins. ${ }^{13}$ GPxs metabolise these lipid hydroperoxides, modulating the production of inflammatory and proaggregatory products e.g. hydroperoxide removal maintains the production of the antiaggregatory prostacyclin while decreasing the production of the pro-aggregatory thromboxane. ${ }^{13}$ (The balance of these two eicosanoids favours blood-clotting in preeclampsia. ${ }^{21}$ ) Overall, the 
effect of the GPxs is to reduce the activity of the cyclooxygenases and lipoxygenases which require a minimum peroxide tone to be optimally active, thus they limit the production of inflammatory and pro-agreggatory prostaglandins and leukotrienes. ${ }^{13}$ Furthermore GPx rapidly reduces peroxynitrite to harmless products. ${ }^{10,11}$ GPx activity was found to be significantly lower in preeclamptic than in normal placental tissue. ${ }^{23}$

Another selenoprotein, thioredoxin reductase (TR), can reduce and detoxify hydrogen peroxide and some lipid peroxides more efficiently than GPxs. ${ }^{24}$ Pre-incubation of an endothelial cell line with physiologically relevant concentrations of selenium protected the cells from oxidative damage by organic peroxides and oxidised LDL through the induction of TR and GPxs. ${ }^{24}$ TR can also reduce dehydroascorbic acid and the ascorbyl free radical back to ascorbate. $^{25}$ Recycling of ascorbate from its oxidized forms is essential to maintain in vivo ascorbate levels $^{25}$ that help to contain oxidative damage to endothelial cells which contain, on a molar basis, more TR than GPxs. ${ }^{24}$

Selenoprotein P may have a special role in the defense of human plasma against peroxynitrite. ${ }^{26}$ It binds to endothelial cells through heparin-binding domains that enable it to coat the surface of cellular membranes, attaching more strongly in the acidotic conditions associated with areas of inflammation. ${ }^{27}$ Its localization to endothelial cells appears to be relevant to its anti-oxidant function which has been demonstrated in the in vitro protection of human plasma from oxidation and nitration mediated by peroxynitrite ${ }^{26}$ and in the protection of the liver from oxidant injury. ${ }^{27}$ Whether a significant antioxidant role for selenoprotein $\mathrm{P}$ in preeclampsia is possible depends on the provision of reducing equivalents in the plasma to regenerate the reduced form of selenoprotein $\mathrm{P}^{26}$, thereby maintaining a catalytic process. However, because selenoprotein P binds to cell membranes, high local concentrations of the 
protein $^{27}$, together with the continuous slow release from cells of the reductant glutathione, may represent a significant source of enzymatic activity. ${ }^{28}$

Selenoprotein P concentrations in plasma are known to be a useful marker of selenium status though neither circulating nor tissue concentrations of selenoprotein P have been measured in human pregnancy. In a UK study, selenoprotein P and plasma GPx together were found to account for up to $90 \%$ of total plasma selenium (cited in reference 26). The greater importance of selenoprotein $\mathrm{P}$, however, is indicated by the fact that its concentration was preserved relative to that of plasma GPx (GPx-3) in subjects reducing their selenium intake. ${ }^{29}$ Relatively low selenium status prior to pregnancy (as measured by toenail selenium concentration) may adversely affect the functional activity of the selenoproteins, reducing their protective effect against oxidative stress. This situation may be exacerbated by the expansion in plasma volume and reduced plasma selenium concentration normally associated with pregnancy. ${ }^{21}$

Implications. Given the serious nature of preeclampsia and the potentially adverse consequences of premature delivery and the associated low birthweight, our results may have important public-health implications, particularly in the light of the reduction in selenium intake and status, both in the UK and in a number of other European countries, over the last 20 to 25 years. ${ }^{13}$ Plasma selenium concentrations are now below the optimum for plasma GPx activity, generally taken to indicate selenium repletion, in a considerable number of European locations. ${ }^{13}$

This study raises the question of whether a small increase in selenium intake might help prevent preeclampsia in susceptible women. We plan to test the hypothesis that adequate selenium status is important for antioxidant defense and peroxynitrite scavenging in pregnant women at risk of preeclampsia, by carrying out a randomized controlled trial of low-dose selenium supplements during pregnancy. 


\section{ACKNOWLEDGEMENTS}

Our thanks are due to research midwives Beth Truelove, Carol Simms and Hazel Coburn of the John Radcliffe Hospital, who assiduously collected the toenail samples over a period of four years, to Jill Adair who prepared the toenail samples for analysis, to Mehmet Sarilar of the Interfaculty Reactor Unit, TU Delft, who carried out the neutron activation analyses, to John Rayman who carried out the data analysis and to Peter Williams for statistical advice. 


\section{REFERENCES}

1. Redman CWG, Sargent IL. Placental debris, oxidative stress and pre-eclampsia. Placenta 2000;21:597-602.

2. Roberts JM, Taylor RN, Musci TJ, Rodgers GM, Hubel CA, and McLaughlin MK. Preeclampsia: an endothelial cell disorder. Am J Obstet Gynecol 1989;161:1200-1204.

3. Redman CWG, Sacks GP, Sargent IL. Preeclampsia: an excessive maternal inflammatory response to pregnancy. Am J Obstet Gynecol 1999;180:499-506.

4. Hubel CA. Oxidative stress in the pathogenesis of preeclampsia. Proc Soc Exp Biol Med 1999;222:222-235.

5. Chappell LC, Seed PT, Briley AL, Kelly FJ, Lee R, Hunt B, et al. Effect of antioxidants on the occurrence of pre-eclampsia in women at increased risk: a randomised trial. Lancet 1999;354:810-816.

6. Myatt L, Rosenfield RB, Eis AL, Brockman DE, Greer I, Lyall F. Nitrotyrosine residues in placenta. Evidence for peroxynitrite formation and action. Hypertension 1996;28:488-93.

7. Kossejans W, Eis A, Sahay R, Brockman D, Myatt L. Role of peroxynitrite in altered fetalplacental vascular reactivity in diabetes or preeclampsia. Am J Physiol Heart Circ Physiol 2000;278:H1311-1319.

8. Roggensack AM, Zhang Y, Davidge ST. Evidence for peroxynitrite formation in the vasculature of women with preeclampsia. Hypertension 1999; 33: 83-89.

9. Kooy NW, Royall JA. Agonist-induced peroxynitrite production from endothelial cells. Arch Biochem Biophys 1994;310:352-359.

10. Arteel GE, Briviba K, Sies H. Protection against peroxynitrite. FEBS Lett 1999;445: 226230. 
11. Lowe D. Nitric oxide dysfunction in the pathophysiology of preeclampsia. Nitric Oxide-Biol Chem 2000;4:441-458.

12. Wolin MS. Interactions of oxidants with vascular signaling systems. Arterioscler Thromb Vasc Biol 2000;20:1430-42.

13. Rayman MP. The importance of selenium to human health. Lancet 2000;356:233-241.

14. Rayman MP, Abou-Shakra FR, Redman CWG, Ward NI. Serum elemental concentrations in the pregnancy disease pre-eclampsia. In: Fischer PWF, L’Abbe M, Cockell KA, Gibson RS, editors. Proceedings of the 9th International Symposium on Trace Elements in Man and Animals. Ottawa, Canada: N. R. C. Research Press; 1997. p. 71-73.

15. Longnecker MP, Stampfer MJ, Morris JS, Spate V, Baskett C, Mason M, et al. A 1-year trial of the effect of high selenium bread on selenium concentrations in blood and toenails. Am $\mathrm{J}$ Clin Nutr 1993; 57:408-413.

16. van den Brandt PA, Goldbohm RA, van't Veer P, Bode P, Hermus RJ, Sturmans F. Predictors of toenail selenium levels in men and women. Cancer Epidemiol Biomarkers Prev 1993;2:107-112.

17. Garland M, Morris JS, Rosner BA Stampfer MJ, Spate VL, Baskett CJ, et al. Toenail trace element levels as biomarkers: reproducibility over a 6-year period. Cancer Epidemiol Biomarkers Prev 1993;2:493-497.

18. Activation analysis of hair as an indicator of contamination of man by environmental trace element pollutants. International Atomic Energy Agency, Report IAEA/RL/50. Vienna: International Atomic Energy Agency; 1978.

19. Redman CWG. Hypertension in pregnancy. In de Swiet M, editor. Medical Disorders of Pregnancy, $4^{\text {th }}$ ed. London: Blackwell Scientific Publications; 2002. 
20. Han L, Zhou S. Selenium supplement in the prevention of pregnancy induced hypertension. Chinese Med J 1994;107:870-871.

21. Rayman MP, Abou-Shakra FR, Ward NI, Redman CWG. Comparison of selenium levels in pre-eclamptic and normal pregnancies. Biol Trace Element Res 1996;55:9-20.

22. McKenzie RC, Rafferty TS. Effects of selenium on immunity and ageing. In Hatfield DL, editor. Selenium: Its Molecular Biology and Role in Human Health. Dordrecht, The Netherlands: Kluwer Academic Publishers; 2001. Chapter 21.

23. Walsh SW, Wang Y. Deficient glutathione peroxidase activity in preeclampsia is associated with increased placental production of thromboxane and lipid peroxides. Am J Obstet Gynecol 1993;169:1456-1461.

24. Lewin MH, Arthur JR, Riemersma RA, Nicol F, Walker SW, Millar EM, Howie AF, Beckett GJ. Selenium supplementation acting through the induction of thioredoxin reductase and glutathione peroxidase protects the human endothelial cell line EAhy926 from damage by lipid hydroperoxides. Biochim Biophys Acta 2002; 1593:85-92.

25. May JM, Mendiratta S, Hill KE, Burk RF. Reduction of dehydroascorbate to ascorbate by the selenoenzyme thioredoxin reductase. J Biol Chem 1997;272:22607-22610.

26. Arteel GE, Mostert V, Oubrahim H, Briviba K, Abel J, Sies H. Protection by selenoprotein P in human plasma against peroxynitrite-mediated oxidation and nitration. Biol Chem 1998;379:1201-1205.

27. Burk RF, Hill KE, Boeglin ME, Ebner FF, Chittum HS. Selenoprotein P associates with endothelial cells in rat tissues. Histochem Cell Biol 1997;108:11-15.

28. Saito Y, Hayashi T, Tanaka A, Watanabe Y, Suzuki M. Selenoprotein P in human plasma as an extracellular phospholipid glutathione peroxidase. J Biol Chem. 1999;274:2866-2871. 
29. Persson-Moschos M, Huang W, Srikumar TS, Åkesson B. Selenoprotein P in serum as a biochemical marker of selenium status. Analyst. 1995;120:833-836. 


\section{FIGURE LEGEND}

Fig. Distribution of toenail selenium concentrations in pre-eclamptic and control subjects.

Horizontal bars represent median values. 
Table I. Clinical characteristics of patient groups

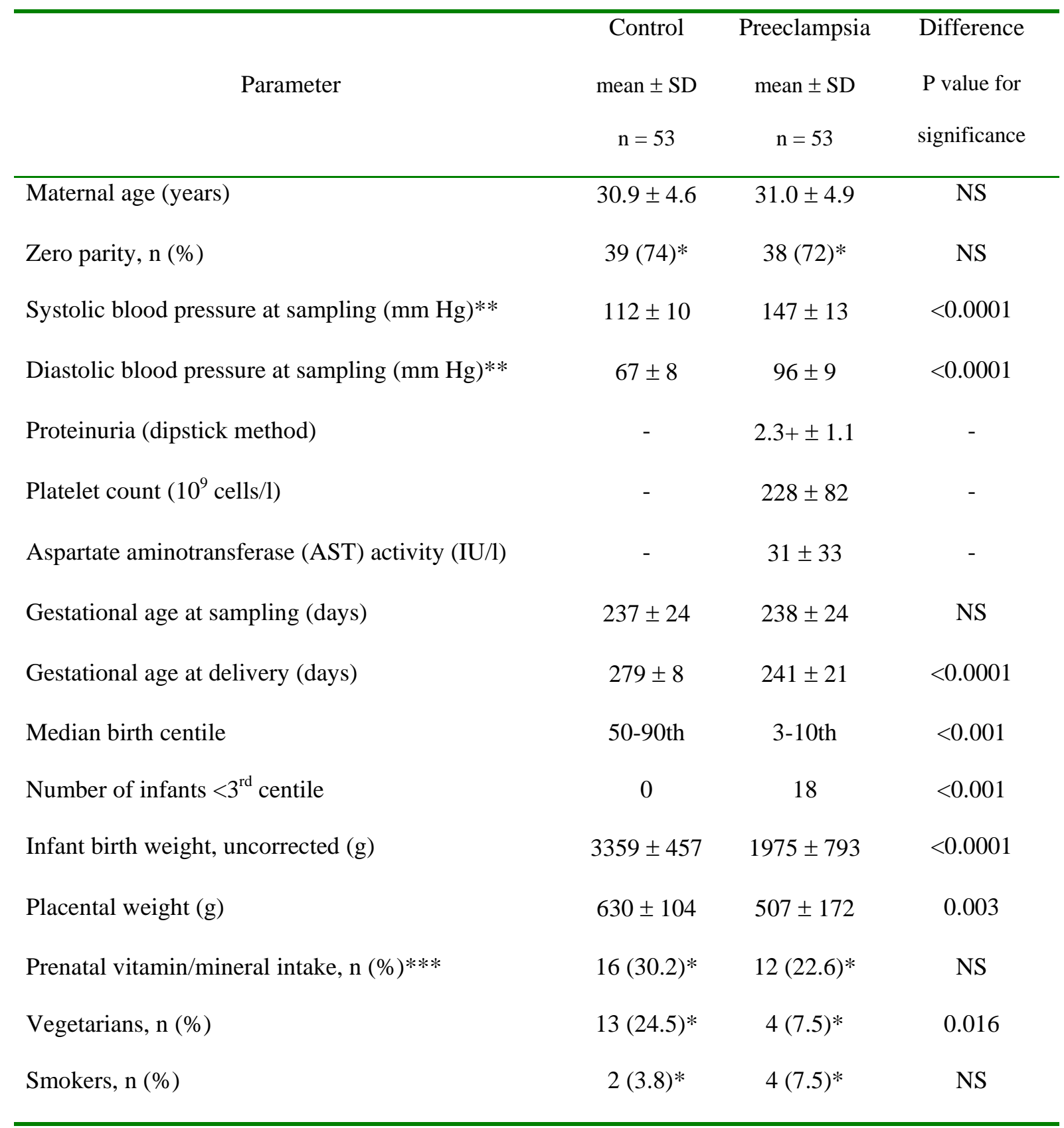

$P$ values calculated from paired-sample $t$ test, Mann-Whitney $\mathrm{U}$ test, $\chi^{2}$ test or Fisher's Exact test as appropriate.

NS, Not significant

*Number (\%)

**66\% of pre-eclamptic subjects received anti-hypertensive medication (methyldopa and/or nifedipine)

***excluding folic acid and iron 
Table II. Distribution of subjects in preeclamptic and control groups by tertile of toenail selenium

\begin{tabular}{ccccc}
\hline Group & Number of & Low Se & Med Se & High Se \\
& subjects & $0.492 \mathrm{mg} / \mathrm{kg}^{*}$ & $0.588 \mathrm{mg} / \mathrm{kg}^{*}$ & $0.707 \mathrm{mg} / \mathrm{kg}^{*}$ \\
\hline Pre-eclamptics & 53 & 26 & 13 & 14 \\
Controls & 53 & 9 & 22 & 22 \\
\hline
\end{tabular}

*Mean toenail selenium concentration 
Table III. Case-control pair status by low vs. medium/high selenium tertile, and odds ratio (OR) and 95\% confidence interval (CI) for pre-eclampsia by lowest tertile vs. rest

\begin{tabular}{lc|cc}
\hline & \multicolumn{2}{|l}{ Control } & \\
& & Low Se tertile & \\
\hline Case & Low Se tertile & Medium/High Se tertile \\
& & 4 & 22 \\
Medium/High Se tertile & 5 & 22 \\
\hline
\end{tabular}

* mean toenail selenium concentration $0.492 \mathrm{mg} / \mathrm{kg}$

OR (lowest tertile vs. rest) $22 / 5=4.4$; $95 \%$ C.I $1.6-14.9$ 
Optional table Table IV. Toenail selenium concentrations in women from other studies

\begin{tabular}{|c|c|c|c|c|c|}
\hline \multirow[t]{2}{*}{ Country } & \multirow{2}{*}{$\begin{array}{l}\text { Number of } \\
\text { Subjects }\end{array}$} & \multirow{2}{*}{$\begin{array}{l}\text { Subjects (age) } \\
\text { Smokers/non- } \\
\text { smokers }\end{array}$} & \multicolumn{2}{|c|}{ Se concentration (mg/kg) } & \multirow[t]{2}{*}{ Reference } \\
\hline & & & Mean \pm SD & Median & \\
\hline \multirow[t]{2}{*}{ UK } & 53 & Pregnant controls & $0.63 \pm 0.09$ & 0.62 & This study \\
\hline & 53 & Pre-eclamptics & $0.57 \pm 0.10$ & 0.56 & \\
\hline \multirow[t]{4}{*}{ Netherlands } & 77 & All subj. (55-70) & $0.693 \pm 0.159$ & 0.679 & van Noord et al. Breast Cancer \\
\hline & & Smokers & 0.65 & - & Res Treat \\
\hline & & Non-smokers & 0.71 & - & 25: 11-19, 1993 \\
\hline & 270 & All subjects & $0.721 \pm 0.220$ & 0.689 & \\
\hline \multirow[t]{3}{*}{ Netherlands } & 1248 & All subjects & $0.575 \pm 0.109$ & - & van den Brandt et al. \\
\hline & & Smokers & $0.548 \pm 0.101$ & - & Cancer Epidemiol Biomarkers \\
\hline & & Non-smokers & $0.581 \pm 0.109$ & - & Prev 2:107-112, 1993 \\
\hline \multirow[t]{2}{*}{ Netherlands } & 1248 & All subjects & $0.575 \pm 0.109$ & - & van den Brandt et al. Cancer Res \\
\hline & & & & & 53:4860-5, 1993 \\
\hline \multirow[t]{2}{*}{ Netherlands } & 1248 & All subjects & $0.575 \pm 0.109$ & - & van den Brandt et al. Am J \\
\hline & & Aged 55-69 & & & Epidemiol 140:20-26, 1994 \\
\hline \multirow[t]{3}{*}{ USA } & 503 & All subj. (36-61) & $0.864 \pm 0.257$ & 0.833 & Garland et al. J Natl Cancer Inst \\
\hline & 144 & Smokers & 0.78 & - & $\mathbf{8 7 : 4 9 7 - 5 0 5 , ~} 1995$ \\
\hline & 258 & Never smokers & 0.85 & - & \\
\hline \multirow[t]{2}{*}{ USA } & 127 & All subj. yr 1982 & $0.83 \pm 0.16$ & 0.81 & Garland et al. Cancer Epidemiol \\
\hline & & All subj. yr 1988 & $0.92 \pm 0.15$ & 0.89 & Biomarkers Prev 2:493-7, 1993 \\
\hline USA & 434 & All subjects & $0.821 \pm 0.174$ & - & Hunter et al. JAMA 264:1128-31, \\
\hline
\end{tabular}


1990

\begin{tabular}{|c|c|c|c|c|c|}
\hline \multirow[t]{4}{*}{ USA } & 96 & All subjects & $0.748 \pm 0.149$ & - & Hunter et al. Am J Epidemiol \\
\hline & 659 & All subjects & $0.801 \pm 0.148$ & - & 132:114-22, 1990 \\
\hline & 146 & Smokers & $0.746 \pm 0.124$ & - & \\
\hline & 311 & Never smokers & $0.817 \pm 0.159$ & - & \\
\hline
\end{tabular}

Table V. (contd) Toenail selenium concentrations in women from other studies 


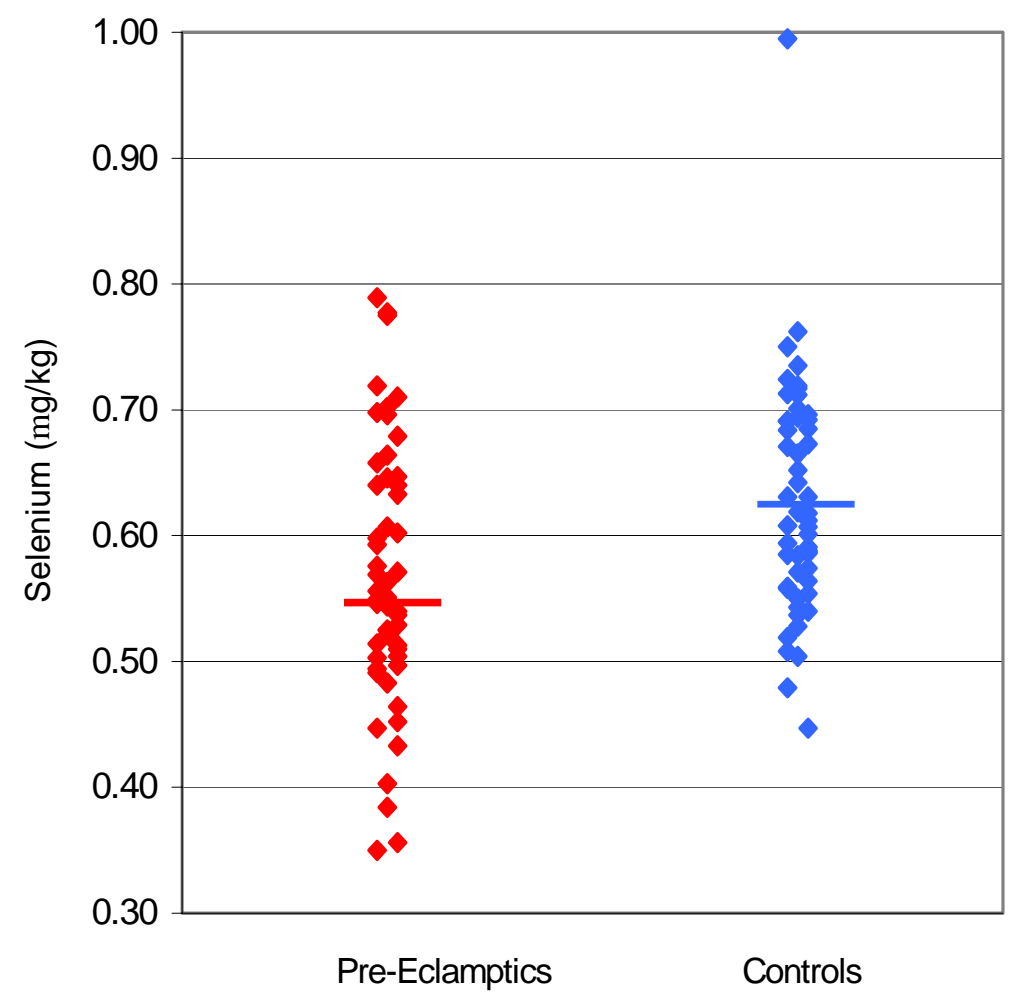

